\title{
Relationship between thyroid disorder and iron deficiency anaemia in pregnancy
}

\author{
Mili D. Chowdhury, S.S. Ghose, Abhish Mohan
}

\begin{abstract}
Corresponding author: Dr. Sivaji S. Ghose, Classified specialist, Department of Surgery, Naval Hospital Powai, Mumbai, Maharashtra-400078, India; Email : sharkdoctor007@gmail.com
\end{abstract}

Distributed under Attribution-Non Commercial - Share Alike 4.0 International (CC BY-NC-SA 4.0)

\begin{abstract}
Background: Thyroid disorders and iron deficiency (ID) are associated with obstetrical and fetal complications. Iron is essential for the normal functioning of thyroid peroxidase (TPO-abs) and ID is frequent during pregnancy. Objective: The present study was undertaken to see the relationship between iron deficiency anaemia and thyroid disorder in antenatal cases. Methods: A total of 100 cases attending the outpatient department of Obstetrics and Gynecology in their 1st trimester (from LMP to 12 weeks) were studied at Naval Hospital Powai during a period from Jan 2016 to Dec 2017. A detailed clinical and obstetric history was taken and patients were subjected to routine antenatal investigations along with thyroid profile. Results: Among 100 cases, 21 antenatal cases had increased TSH. Out of which 4 cases had overt hypothyroidism and 17 cases had subclinical hypothyroidism. 2 cases had decreased thyroid stimulating hormone in early trimester out of which only 1 had overt hyperthyroidism. In the same study 23 cases had mild iron deficiency anaemia and 4 had moderate anaemia. It was later correlated that out of 27 cases with anaemia 14 had hypothyroidism. 51\% of the cases with anaemia had hypothyroidism and $60 \%$ of the hypothyroid cases had iron deficiency anaemia. Applying regression analysis between TSH and Hb level, there was a positive correlation between these two factors in cases. $r^{2}$ value was found to be 0.2871 . This was statistically significant with a $\mathrm{P}$ value of less than 0.005 . Conclusion: It is concluded that there is increased prevalence of thyroid disorders and iron deficiency anaemia in antenatal cases and there is relationship between iron deficiency anaemia and hypothyroid cases.
\end{abstract}

Keywords: Thyroid disorders, iron, antenatal, anaemia, hypothyroidism, hyperthyroidism.

Pregnancy results in progressive anatomical, physiological and biochemical changes affecting all systems of the body. This is principally a phenomenon of maternal adaption to the increasing demands of the growing fetus. Thyroid disorders are the second most common endocrine disorders after diabetes mellitus affecting women in their reproductive age ${ }^{1}$. To understand abnormal thyroid function in pregnancy a quick revision of the physiological changes are warranted. Thyrotropin releasing hormone (TRH) is secreted by hypothalamus and stimulate thyrotropic cells of anterior pituitary to release thyroid stimulating hormone $(\mathrm{TSH})^{2}$. In mother there is marked increase in hepatic production of thyroxine binding globulin due to estrogen stimulation, low metabolism and placental production of human chorionic gonadotrophin. HCG has thyrotropin like activity and stimulates free $T_{4}$ secretion. This in turn inhibits maternal secretion of thyrotropin ${ }^{2}$.

The thyroid gland increases production of thyroid hormones by 40 to $100 \%$ to meet maternal and fetal needs. There is thyroid enlargement because of glandular

Received: $7^{\text {th }}$ April 2020, Peer review completed: $22^{\text {th }}$ May 2020, Accepted: $4^{\text {th }}$ June 2020.

Chowdhury MD, Ghose SS, Mohan A. Relationship between thyroid disorder and iron deficiency anaemia in pregnancy. The New Indian Journal of OBGYN. 2021; 7(2): 163-67. 
The New Indian Journal of OBGYN. 2021 (January-June);7(2)

hyperplasia and increased vascularity. Approximately a third of women experiences hypothyroxinemia. Fetal thyroid does not begin to concentrate iodine until 10-12 weeks of gestation and secretion of thyroid hormone by fetal pituitary ensues at $20 \mathrm{wks}$. Till then fetus is dependent on hormones supplied from mother ${ }^{3}$. Early exposure of thyroid hormone is needed for development of fetal nervous system ${ }^{2}$. Despite several public health programs severe iodine deficiency affects more than 2 million people globally. Uncontrolled thyrotoxicosis and untreated hypothyroidism are associated with adverse pregnancy outcomes. Most thyroid disorders are linked to auto antibodies against thyrocytes. Thyroid peroxidase antibodies have been identified in $5-15 \%$ of pregnant women and these antibodies are associated with early pregnancy loss and preterm birth ${ }^{4}$. Incidence of thyrotoxicosis in pregnancy varies from 2 and 17 per 1000 births ${ }^{2,5}$ whereas incidence of hypothyroidism in India is $4.8-11 \%{ }^{6}$.

The hypervolemia associated with normal pregnancy averages $40-45 \%$ above non pregnant blood volume after 3234 weeks of gestation ${ }^{7}$. By $12 \mathrm{wks}$ plasma volume expands by $15 \%$ compared with that value prior to pregnancy. The number of iron rich oxygen carrying red blood cells swells by $25 \%$ to supply the growing fetus. There is elevated plasma erythropoietin. Because of plasma augmentation, hemoglobin concentration and hematocrit declines. Total iron content is $2-2.5 \mathrm{gm}$ and iron stores are $300 \mathrm{mg}$. As a result woman's body needs twice as much iron and hence iron deficiency anemia is one of the most common deficiency diseases ${ }^{8,9}$. Lower iron level is partly due to menstrual blood loss, deficiency of hepcidin (a peptide hormone which function as homeostatic regulator of systemic iron metabolism). Hepcidin increases with inflammation but decreases with anemia, hormones like estrogen, testosterone, vitamin $\mathrm{D}$, prolactin etc ${ }^{10}$. The prevalence of pregnancy related iron deficiency anemia in India is $75 \%$ and in developed countries is $14 \%{ }^{11}$. Iron deficiency anemia and untreated thyroid disorders in pregnancy are proven risk factors. Iron deficiency anemia is related to hypothyroidism and can be explained by autoimmunity, relation of hepcidin and some maternal hormones ${ }^{12}$.

The present study was carried out at NH Powai in the Department of Obstetrics and Gynecology form Jan 2016 to Dec 2017 for a period of almost 2 years to find the incidence of thyroid disorders and iron deficiency anemia in pregnancy and also find any association between thyroid disorder and iron deficiency anemia in antenatal cases.

\section{Material and methods}

The present study was conducted in 100 antenatal cases who attended the OPD in their first trimester of pregnancy i.e. before $12 \mathrm{wks}$ of gestation and parity up to three in the department of Obstetrics and Gynecology at Naval Hospital Powai for a period of 2 years from January 2016 to December 2017. Patients diagnosed with thyroid disorder and were on medication, cases with severe anemia and cases who attended OPD and were residing outside Maharashtra were excluded from the study.

A detailed history included patients particular, personal, family, menstrual and obstetric history of the patients in the proforma was obtained along with general, systemic and obstetrical examination findings. After proper counselling the cases were asked to undergo routine antenatal investigations along with thyroid profile included serum $\mathrm{TSH}$, free T3, free T4. In the first visit i.e in the first trimester all the cases underwent routine antenatal investigations which included complete haemogram i.e RBC, $\mathrm{WBC}, \mathrm{Hb} \%$, Platelet count, $\mathrm{PCV}, \mathrm{MCV}, \mathrm{MCH}, \mathrm{MCHC}$. Along with these thyroid profiles for all the cases including serum TSH, Free T3, Free T4 were done. The cases who have positive findings were diagnosed and treated accordingly in conjunction with Medical Specialist. In positive cases for thyroid disorders anti TPO was done and in cases with low $\mathrm{Hb} \%$ iron study along with peripheral blood smear were done to confirm the type of anemia. Thalassemia was not included in the study. The cases were called for antenatal checkups as per WHO criteria and in high risk cases more frequently. The study group was subjected to investigations as per WHO guidelines. They were followed up till delivery and 6wks postnatal.

In present study we have used trimester specific reference ranges for thyroid tests ${ }^{13}$, reference ranges for iron deficiency anemia ${ }^{14,15}$, hemoglobin cutoff for anemia as per ICMR ${ }^{16,17}$ and reference ranges for various indicators of Iron deficiency anemia ${ }^{15,18}$. The reports of the cases were assembled and compared to see the relationship between thyroid disorder and iron deficiency anemia in pregnancy. In addition to this incidence of thyroid disorder, iron deficiency anemia in pregnancy was calculated. Finally their effects on fetal and maternal outcome were observed. The regression analysis was done between TSH and $\mathrm{Hb}$ level to find the correlation.

\section{Results}

Among 100 antenatal cases, 21 had increased thyroid stimulating hormone (TSH) of which 4 cases were diagnosed 
to have overt hypothyroidism and rest 17 cases had subclinical hypothyroidism. 2 cases had decreased thyroid stimulating hormone in their early trimester, out of which again one had overt hypothyroidism and the second was euthyroid in second trimester (table 1). cases. $\mathrm{r}^{2}$ value was found to be 0.2871 . This was statistically significant with a $\mathrm{P}$ value of less than 0.005 (figure 1).

\section{Discussion}

More than one-third of pregnant women are iron deficient, placing them at increased risk of a thyroid

Table 1: Thyroid function in different trimester

\begin{tabular}{cccccccc} 
Table 1: Thyroid function in different trimester & \multicolumn{1}{l}{} \\
\hline Trimester & $\uparrow$ TSH & $\downarrow$ FT3 & $\downarrow$ FT4 & Anti TPO & $\downarrow$ TSH & $\uparrow$ T3 & $\uparrow$ T4 \\
\hline First trimester & 20 & - & 4 & 4 & 2 & 2 \\
Second trimester & 1 & - & - & - & - & - \\
Third trimester & - & - & - & - & - & - \\
Total & 21 & 00 & 04 & 04 & 02 & 02 & 01 \\
\hline
\end{tabular}
disease that increases the likelihood of pregnancy complications such as

27 cases had iron deficiency anemia, among them 23 had mild anemia and 4 had moderate anemia as shown in table 2 . It was later correlated that out of 27 cases who had iron deficiency 14 (11 mild anaemia +3 moderate anaemia) had hypothyroidism. But again all hypothyroidism patients didn't have iron deficiency anemia. The only case that had hyperthyroidism never had anemia in her antenatal period.

Table 2: Iron deficiency anaemia with $\mathrm{Hb} \%<11 \mathrm{gm} \%$

\begin{tabular}{ccccc}
\hline Trimester & Severe & Moderate & Mild & Total \\
\hline First trimester & - & 2 & 12 & 14 \\
Second trimester & - & 1 & 8 & 9 \\
Third trimester & - & 1 & 3 & 4 \\
Total & - & 04 & 23 & 27 \\
\hline
\end{tabular}

Table 3 shows the correlation between thyroid dysfunction and Iron deficiency anaemia.

Table 3: Correlation between thyroid dysfunction and iron deficiency anaemia

\begin{tabular}{ccc}
\hline Iron deficiency anemia & Hypothyroidism & Hyperthyroidism \\
\hline Mild Anaemia & 11 & - \\
Moderate Anaemia & 3 & - \\
Severe Anaemia & - & 1 \\
\hline
\end{tabular}

miscarriage, placental abruption growth restriction ${ }^{19}$. Poppe et al reported that $35 \%$ of almost 2000 pregnant women had iron deficiency during the first trimester, and that this increased the risk of thyroid autoimmunity by over $50 \%{ }^{20}$. While previous studies have indicated that iron deficiency during pregnancy can affect from $24 \%$ to $44 \%$ of women, this is the first to show the secondary effect of an increased prevalence of thyroid autoimmunity ${ }^{21}$. However, autoimmune thyroiditis contributes towards hypothyroidism in pregnancy in India is 4.8-12\%. Incidence of hypothyroidism in women with recurrent pregnancy loss is about 4-16\%. Hence Indian Thyroid Society recommends screening of thyroid stimulating hormone in all pregnant women at time of first visit and treatment to be started if $\mathrm{TSH} 2.5 \mathrm{IU} / \mathrm{ml}^{22}$. ACOG recommends thyroid screening in women with history or symptoms of thyroid disease and doesn't recommend universal In the present study, 21 antenatal cases had increased thyroid stimulating hormone (TSH). Out of which 4 cases were diagnosed to have overt hypothyroidism and rest 17 cases had subclinical hypothyroidism. 2 cases had decreased thyroid hormone (table 4 ).

In following up the cases during post natal (4-6 wks) period especially the cases with abnormal thyroid function in

Table 4: Thyroid dysfunction and iron deficiency anemia

\begin{tabular}{ccccc}
\hline $\begin{array}{c}\text { Iron deficiency } \\
\text { anemia }\end{array}$ & Hyperthyroidism & Hypothyroidism & $\begin{array}{c}\text { Subclinical } \\
\text { hyperthyroidism }\end{array}$ & $\begin{array}{c}\text { Subclinical } \\
\text { hypothyroidism }\end{array}$ \\
\cline { 2 - 5 } & - & 4 & - & 5 \\
Mild anaemia & - & 2 & - & 3 \\
Moderate anaemia & 1 & - & 1 & 9 \\
Severe anaemia & & \multicolumn{4}{c}{ Thyroid dysfunction } \\
\hline
\end{tabular}
screening ${ }^{23}$. Endocrine society 2012 and WHO has no recommendation for universal thyroid screening in pregnancy but some studies stated that limiting screening to high risk individuals will miss $30 \%$ women with overt or subclinical hypothyroidism ${ }^{24}$.

The prevalence of anemia in overt hypothyroidism is $43 \%$ and $39 \%$ in subclinical hypothyroidism ${ }^{25}$. Growing researches suggests that iron deficiency with or without anemia impairs thyroid metabolism by decreasing plasma $\mathrm{T} 3$ and $\mathrm{T} 4$, reducing peripheral conversation of $\mathrm{T} 4$ to $\mathrm{T} 3$ and increasing $\mathrm{TSH}$ their antenatal period and were on treatment, it was found that 21 cases were euthyroid with medicine and 2 cases had raised TSH and was found that both had left medicine on their own. One case had decreased FT4. In the present study maternal ad fetal outcome were favorable.

Applying regression analysis between TSH and $\mathrm{Hb}$ level, there was a positive correlation between these two factors in concentration ${ }^{26}$. Iron deficiency anemia affects thyroid metabolism by inducing alteration in common hypothalamo pituitary thyroid axis, reducing T3 binding to hepatic nuclear receptor or through anemia and lower $\mathrm{O} 2$ transport. Iron deficiency anemia impairs the hepatic T4-5 deiodinase, an enzyme which converts T4-T3 ${ }^{27}$ on thyroid peroxidase responsible for thyroid hormone synthesis. Hypothyroidism 
can cause a variety of anemic disorders. Iron deficiency anemia can result from impaired intestinal iron absorption related to thyroid hormone deficiency on gastric achlorhydria.

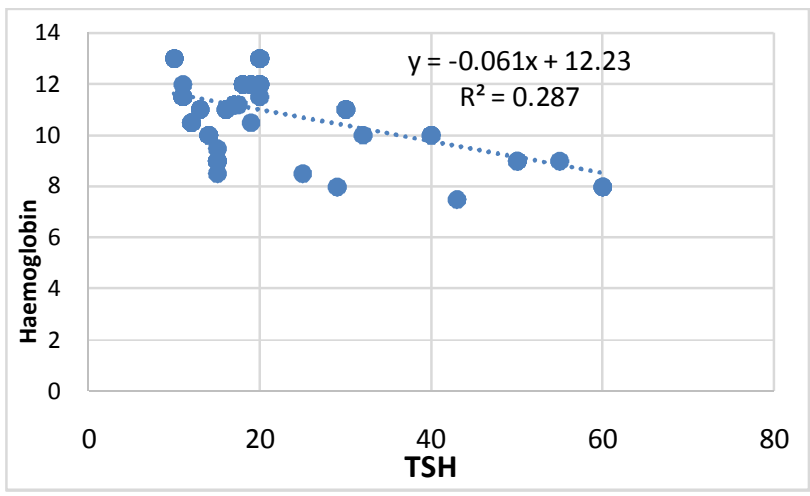

Figure 1: Correlation between TSH and $\mathrm{Hb}$ in cases

The existing study was conducted in hundred pregnant women visiting the obstetrics and gynecology OPD at Naval Hospital Powai in their first trimester. Out of 100, 21 cases had increased thyroid stimulating hormones. Out of which 4 cases were overt hypothyroid and rest 17 cases had subclinical hypothyroidism. Hence prevalence of hypothyroidism in pregnancy was $0.21 \%(21 \%)$ in our study. Prevalence of overt hypothyroidism was $0.4 \%$ (4\%) and subclinical hypothyroidism was $0.17 \%(17 \%)$ in pregnancy. These findings are compared with a study done by Dhanwal et al ${ }^{28}$; they reported prevalence of hypothyroidism in pregnancy was $13.13 \%$ and major being subclinical. In a study by Agarwal et al ${ }^{29}$, the prevalence was $31.8 \%$. Our findings are more or less correlated with the above studies. Slightly higher prevalence rate may be due to following reasons: $9 \%$ of the cases in the study group were infertile and most of them who were positive for thyroid dysfunction were north Indians and from Odisha. Prevalence of hypothyroidism in our study was $1 \%$ which is correlated with the findings of Indian Thyroid Society ${ }^{22}$.

In existing study 27 cases had iron deficiency anemia and hence the prevalence becomes $27 \%$ compared to nutrition impact model study, estimated global prevalence of anemia in pregnancy is $38 \%$ in 2011 with range varying from $17 \%$ in developed and $56.4 \%$ in developing countries. Current study has a lesser prevalence because of better nutritional status of the cases being studied. Out of 27 cases who had iron deficiency anemia, 14 cases had hypothyroidism i.e $50 \%$ of the cases with anemia had hypothyroidism and $60 \%$ of the hypothyroid cases had iron deficiency anemia which is comparable with the study done by Shuxiang et al ${ }^{26}$.

\section{Conclusion}

From the results of present study, it is concluded that there is increased prevalence of thyroid disorders and iron deficiency anaemia in antenatal cases and there is relationship between iron deficiency anaemia and hypothyroid cases.

\section{Conflict of interest: None. Disclaimer: Nil.}

\section{References}

1. Carney LA, Quinlan JD, West JM. Thyroid disease in pregnancy. Am Fam Physician. 2014; 89(4): 273-8.

2. Sreelatha S, Kamala R, Nadagoudar S, Shruthi A, Bharathi A. A clinical review of obstetric and perinatal outcome in thyroid disorders. Endocrinol Metab Int J. 2018; 6(4): 266-82.

3. Alemu A, Terefe B, Abebe M, Biadgo B. Thyroid hormone dysfunction during pregnancy: A review. Int J Reprod Biomed (Yazd). 2016;14(11): 677-86.

4. Abbassi-Ghanavati M, Casey BM, Spong CY, McIntire DD, Halvorson LM, Cunningham FG. Pregnancy outcomes in women with thyroid peroxidase antibodies. Obstetrics and Gynecology. 2010;116(2 Pt 1):381-6.

5. Mestman JH. Hyperthyroidism in pregnancy. Clin Obstet Gynecol. 1997; 40(1): 45-64.

6. Nambiar V, Jagtap VS, Sarathi V, Lila AR, KamalanathanS, BanlgarTR, et al. Prevalence and impact of thyroid disorder or maternal outcome in Asian-Indian pregnant women. J Thyroid Res. 2011; (42): 90-7.

7. Akinlaja O. Hematological Changes in Pregnancy - The Preparation for Intrapartum Blood Loss. Obstet Gynecol Int J. 2016; 4(3): 00109.

8. Morrison JC, Parrish MR. Anemia Associated with Pregnancy. Glob. libr. women's med. 2016; DOI 10.3843/GLOWM.10164.

9. Adam I, Ali AA. Anemia during pregnancy. Nutritional deficiency, InTech ;2016. DOI: 10.5772/63211

10. Sangkhae V, Nemeth E. Regulation of the Iron Homeostatic Hormone Hepcidin. Adv Nutr. 2017; 8(1):126-36.

11. Kalaivani K. Prevalence \& consequences of anaemia in pregnancy. Indian J Med Res. 2009;130(5): 627-33. 
12. Ahmad N, Kalakoti P, Bano R, Syed MMA. The prevalence of anaemia and associated factors in pregnant women in a rural Indian community. AMJ. 2010; 3(5):276-80.

13. ACOG. Practice Bulletin No. 148: Thyroid Disease in Pregnancy. Obstet Gynecol. 2015 Apr;125(4):996-1005.

14. WHO. The global Prevalence of anaemia 2011. Geneva: WHO; 2015.

15. FOGSI. FOGSI General Clinical Practice Recommendation, Management of Iron Deficiency Anaemia in pregnancy. Mumbai: FOGSI; 2017.

16. Indian Council of Medical Research. Evaluation of National Nutritional Anaemia Prophylaxis Programme. Task Force study. New Delhi: ICMR; 1989.

17. Sharma JB, Shankar M. Anaemia in Pregnancy. Journal of International Medical Sciences Academy. 2010; 23 (4): 253-60.

18. Burke RM, Leon JS, Suchdev PS. Identification, Prevention and Treatment of Iron Deficiency during the First 1000 Days. Nutirents. 2014; 6: 4093-114.

19. Tingi E, Syed AA, Kyriacou A, Mastorakos G, Kyriacou A. Benign thyroid disease in pregnancy: A state of the art review. J Clin Transl Endocrinol. 2016; 6: 37-49.

20. Belhomme J, Rozenberg S, Pepersack T, Poppe K. Prevalence of thyroid autoimmunity and dysfunction in women with iron deficiency during early pregnancy: is it altered? European Journal of Endocrinology. 2016; 175(3):191-9.

21. Veltri F, Poppe K. Variables contributing to thyroid (Dys) function in pregnant women: more than thyroid antibodies? Eur Thyroid J. 2018; 7(3):1-9.

22. Ministry of Health and Family Welfare. National Guidelines of screening hypothyroidism in Pregnancy. New Delhi: Maternal Health Division, Ministry of Health \& Family welfare GOI; Dec 2014.
23. American College of Obst and Gynaecology. Practice bulletin No 37. Washington DC: Am College of Obst \& Gynaecology; 2002.

24. Vaidya B, Anthony S, Bilous M, Shields B, Drury $\mathrm{J}$, Hutchison S, et al. Detection of thyroid dysfunction in early pregnancy: universal screening or targeted highrisk case finding? The Journal of Clinical Endocrinology and Metabolism. 2007; 92(1): 203-7.

25. Erdogan M, Kosenli A, Ganidagli S, Koluksizoglu M. Characteristics of anaemia in subclinical and overt hypothyroid patient. Endocrinol Journal. Dec 2011; 59(3):213-20.

26. Shuxiang L, Xin GAO, Yancai WE. The relationship between IDA and thyroid function in Chinese women during early pregnancy. Journal of Nutritional Science and Vitaminology. 2016; 62(6): 397-401.

27. Hess SY, Zimmermann MB, Arnold M, Langhans W, Hurrell RF. Iron definition anaemia reduces thyroid peroxidase activity. J Nutr 2002;132: 1951-5.

28. Dhanwal DK, Bajaj S, Rajput R, Subramaniam K, Chowdhury S, Bhandari R. Prevalence of hypothyroidism in pregnancy: An epidemiological study from 11 cities in 9 states of India. Indian J Endocrinol Metab. 2016;20(3):387-90.

29. Agrawal U, Shrivastava P, Shrivastava S. Hypothyroidism and anemia in pregnancy. Int $\mathrm{J}$ Adv Med 2016; 3: 851-4.

Mili D. Chowdhury ${ }^{1}$, S.S. Ghose ${ }^{2}$, Abhish Mohan ${ }^{3}$

${ }^{1}$ Graded specialist, Department of Obstetrics \& Gynaecology, Naval Hospital Powai, Mumbai, Maharashtra, India; ${ }^{2}$ Classified specialist, Department of Surgery, Naval Hospital Powai, Mumbai, Maharashtra, India; ${ }^{3}$ Graded specialist, Department of Medicine, Naval Hospital Powai, Mumbai, Maharashtra, India. 